\title{
Scheduling without payments
}

\author{
Elias Koutsoupias*
}

July 3, 2011

\begin{abstract}
We consider mechanisms without payments for the problem of scheduling unrelated machines. Specifically, we consider truthful in expectation randomized mechanisms under the assumption that a machine (player) is bound by its reports: when a machine lies and reports value $\tilde{t}_{i j}$ for a task instead of the actual one $t_{i j}$, it will execute for time $\tilde{t}_{i j}$ if it gets the task-unless the declared value $\tilde{t}_{i j}$ is less than the actual value $t_{i j}$, in which case, it will execute for time $t_{i j}$. Our main technical result is an optimal mechanism for one task and $n$ players which has approximation ratio $(n+1) / 2$. We also provide a matching lower bound, showing that no other truthful mechanism can achieve a better approximation ratio. This immediately gives an approximation ratio of $(n+1) / 2$ and $n(n+1) / 2$ for social cost and makespan minimization, respectively, for any number of tasks.
\end{abstract}

\section{Introduction}

A major challenge today is to design algorithms that work well even when the input is reported by selfish agents or when the algorithm runs on a system with selfish components. The classical approach is to use mechanism design [13], that is, to design algorithms that use payments to convince the selfish agents to reveal the truth and then compute the outcome using the reported values. Central to the mechanism design approach is the use of dominant strategies as the equilibrium concept. Mechanism design is a very important framework with many unexpected results and it remains a very active research area trying to address some beautiful and challenging problems. Nevertheless, one major problem with mechanism design with payments is that in many situations, the use of payments may not be feasible.

Partly for this reason, there is a lot of recent interest in mechanisms that use no payments (mechanism design without payments) [13]. Given that in many problems we have obtained very poor results using mechanisms with payments, it will be really surprising if the substantially more restricted class of mechanisms without payments can achieve any positive results. For the general unrestricted domain with at least 3 outcomes and truthful mechanisms without payments, the Gibbard-Satterthwaite theorem [6, 17] states that only dictatorial mechanisms are truthful; dictatorial mechanisms are those in which a particular player determines the outcome. Contrast this

*Department of Informatics, University of Athens. Email: elias@di .uoa.gr 
to Roberts theorem [16], which states that if we allow payments, the truthful mechanisms are the affine maximizers, a much richer class than the dictatorial mechanisms (yet a very poor class in comparison to the set of all possible algorithms).

When we restrict the domain to scheduling unrelated machines, perhaps the most influential problem in algorithmic game theory, the results so far have also been disappointing. The best approximation ratio for the makespan that we know by truthful in expectation mechanisms is $(n+1) / 2$ [3], where $n$ is the number of players. The best known lower bound of 2 [3, 11] leaves the possibility open for improved mechanisms. The situation for deterministic mechanisms is similar: the upper bound is easily $n$ [12], and the best known lower bound is 2.61 [8]. It may seem surprising that in this work we can achieve comparable results using mechanisms without payments (our main result, Theorem 2, is a truthful in expectation mechanism with approximation ratio $n(n+1) / 2$ ). But a moments thought will reveal that we can get a slightly weaker bound (approximation ratio $n$ for one task and hence approximation ratio $n^{2}$ for many tasks) with the natural mechanism which allocates each task independently and with probability inversely proportional to the execution times (Proposition 1). The assumption that the players are bound by their declarations plays also a crucial role; without it, no positive result is possible. The value of our main result is that it gives a definite answer (tight upper and lower bounds, albeit only for one task) for this fundamental problem.

Mechanism design without payments is a major topic in game theory [13], although it has not been studied as intensively as the variant with payments in algorithmic game theory. There are however many recent publications which study such mechanisms. Procaccia and Tennenholtz [15] proposed to study approximate mechanism design without payments for combinatorial problems and they studied facility location problems. This work was substantially extended (see for example [10, 9, 1]). Such mechanisms studied also by Dughmi and Gosh [4] who consider mechanisms assignment problems and by Guo and Conitzer [7] who consider selling items without payments to 2 buyers. Conceptually, closer to our approach of assuming that the players pay their declared values is the notion of impositions of Nissim, Smorodinsky, and Tennenholtz [14] which was further pursued by Fotakis and Tzamos [5].

\section{Model}

We study the problem of scheduling tasks when the machines are selfish. We formulate the problem in its more general form, the unrelated machines version: there are $n$ selfish machines and $m$ tasks; the machines are lazy and prefer not to execute any tasks. Machine $i$ needs time $t_{i, j}$ to execute task $j$. The tasks are allocated to machines with the objective of minimizing the makespan (or the social welfare which is the negation of the sum of executing times of all machines). Let $a$ be an (optimal) allocation for input $t$, where $a_{i, j}$ is an indicator variable about the event of allocating task $j$ to machine $i$. The execution time of machine $i$ is $\sum_{j=1}^{m} a_{i, j} t_{i, j}$ and the makespan is $\max _{i=1, \ldots, n} \sum_{j=1}^{m} a_{i, j} t_{i, j}$. For randomized algorithms, the allocation variables $a_{i, j}$ are not integral but real values in $[0,1]$ which is the probability to allocate task $j$ to ma- 
chine $i_{1}^{1}$

In this work, we consider direct revelation mechanisms; a mechanism is simply a randomized scheduling algorithm $S$ which computes an allocation based on the declarations of the machines. There are no payments. More precisely, every machine $i$ reports its private values $\tilde{t}_{i, j}$, one for each task and we apply the algorithm $S$ on this input. The notation $\tilde{t}_{i, j}$ instead of $t_{i, j}$ is used because the machine may lie and not declare its true values $t_{i, j}$. There is however a very important difference with the standard Nisan-Ronen framework [12] for this mechanism design problem. We assume that the machines are bound by their reports. More precisely, if a machine $i$ declares a value $\tilde{t}_{i, j} \geq t_{i, j}$ for task $i$ and is allocated the task, its actual cost is the declared value $\tilde{t}_{i, j}$ and not $t_{i, j}$. One justification for this assumption is that in some environments the machines can be observed during the execution of the tasks and cannot afford to be caught lying about the execution times. Similar assumptions have been used for other problems. Our assumption is similar to the notion of imposition [14, 5]; for example, in the facility location problem, the players may be forced to use the facility which is closer to their declared position instead of letting them freely choose between the facilities. To complete our assumptions, we need to specify what happens when a machine declares a smaller value, i.e., $\tilde{t}_{i, j}<t_{i, j}$. In this case, we make the simple assumption that the actual cost is the true value $t_{i, j}$; it would be simpler to assume that the machines are not allowed to lie in this direction, but we prefer the weakest assumption since it does not affect our results. To summarize, the cost of machine $i$ for task $j$ is $\max \left(t_{i, j}, \tilde{t}_{i, j}\right)$.

Our framework now is simple. We design a randomized scheduling algorithm $S$. The selfish machines report values $\tilde{t}_{i, j}$ and we apply algorithm $S$ to the reported values. This induces a game among the machines: the pure strategies of machine $i$ are the vectors $\left(\tilde{t}_{i, j}\right)_{j=1}^{m}$ with $\tilde{t}_{i, j} \geq 0$. The cost is the execution time computed by algorithm $S$ on input $\tilde{t}$. To be more precise, if $a=S(\tilde{t})$ is the allocation computed by algorithm $S$ on input $\tilde{t}$, the cost of machine $i$ is $c_{i}=\sum_{j=1}^{m} a_{i, j} \max \left(t_{i, j}, \tilde{t}_{i, j}\right)$. We seek mechanisms which minimize the makespan $\max _{i=1, \ldots, n} c_{i}$ or the social cost $\sum_{i=1}^{n} c_{i}$.

Our assumption is related to mechanisms with verification [12], in which the mechanism learns the actual execution time of the machines and pays after the execution. Because of the delayed payments, these mechanisms are much more powerful than the mechanisms of our framework; for example, they can enforce that the machines are bound by their declarations by imposing a very high penalty for lying. A similar framework was proposed in [2], but in this case the power of the mechanism is limited: it can only deny payment at the end when a machine is caught lying by not being able to finish the tasks within the declared time.

\subsection{The case of a single task}

We focus on the simple case of one task and $n$ machines. Let $t_{1}, \ldots, t_{n}$ be the true values of the machines for the task and let $\tilde{t}_{1}, \ldots, \tilde{t}_{n}$ be the declared values; we drop the second subscript since there is only one task. Let $p_{i}(\tilde{t})$ be the probability of allocating the task to player $i$. The expected cost of player $i$ is $c_{i}=c_{i}\left(t_{i}, \tilde{t}\right)=p_{i}(\tilde{t}) \max \left(t_{i}, \tilde{t}_{i}\right)$, while

\footnotetext{
${ }^{1}$ We may also interpret the probabilities as fractional allocations. Naturally, our results apply to the fractional allocations as well.
} 
the social cost of the algorithm is $\sum_{i=1}^{n} c_{i}$ (in the case of the single task, the makespan and social cost are identical).

The mechanism is called truthful if for every $t_{-i}$, the expected cost $c_{i}$ of player $i$ is minimized when $\tilde{t}_{i}=t_{i}$. This notion of truthfulness, truthful in expectation, is the weakest notion of truthfulness which contains a richer class of mechanisms than the standard notion on truthfulness or universal truthfulness. It is not ex post truthful, meaning that after the players see the outcome of the coins, they may want to change their declarations. It is trivial to see that the stronger notion of truthfulness (universally truthful) cannot achieve any positive result. Notice however, that for the fractional version of the scheduling problem, in which we can allocate parts of the same tasks to different machines, we can consider deterministic algorithms and consequently the strongest notion of truthfulness.

Claim 1. An algorithm is truthful if and only if for every $i$ and $t_{-i}, t_{i} p_{i}(t)$ is nondecreasing in $t_{i}$ and $p_{i}(t)$ is non-increasing in $t_{i}$.

Proof. Indeed, if $t_{i} p_{i}(t)$ is non-decreasing in $t_{i}$, the player prefers $t_{i}$ to higher values, i.e., when $\tilde{t}_{i} \geq t_{i}$, the cost $c_{i}=p_{i}\left(\tilde{t}_{i}, t_{-i}\right) \tilde{t}_{i}$ is minimized at $\tilde{t}_{i}=t_{i}$. Similarly, if $p_{i}\left(t_{i}\right)$ is non-increasing, the player prefers $t_{i}$ to smaller values, i.e., when $\tilde{t}_{i} \leq t_{i}$, the cost $c_{i}=p_{i}\left(\tilde{t}_{i}, t_{-i}\right) t_{i}$ is again minimized at $\tilde{t}_{i}=t_{i}$.

Conversely, if there exist $x<y$ with $p_{i}\left(t_{-i}, y\right) y<p_{i}\left(t_{-i}, x\right) x$, then player $i$ gains by lying: when $t_{i}=x$ he prefers to declare $y$. Similarly, if there exist $x>y$ with $p_{i}\left(t_{-i}, x\right)>$ $p_{i}\left(t_{-i}, y\right)$, the player would again prefer to declare $y$ when $t_{i}=x$.

A mechanism is defined simply by the probability functions $p_{i}(t)$. The expected makespan is $\sum_{i} c_{i}$ and its approximating ratio is $\sum_{i} c_{i} / \min _{i} t_{i}$. We seek truthful mechanisms with small approximation ratio.

\section{Truthful mechanisms for one task}

In this section, we study the case of a single task. We first consider a natural mechanism, the proportional algorithm: It allocates the task to machine $i$ with probability inversely proportional to the declared value $t_{i}$, i.e., $p_{i}(t)=t_{i}^{-1} / \sum_{k} t_{k}^{-1}$.

Proposition 1. The proportional algorithm is truthful and achieves approximation ratio $n$.

Proof. Indeed, to verify that the mechanism is truthful, it suffices to observe that $p_{i}(t)$ is non-increasing in $t_{i}$ and that $t_{i} p_{i}(t)$ is non-decreasing it $t_{i}$. The expected makespan of this mechanism is $n / \sum_{i} t_{i}^{-1}$ while the optimal makespan is $\min _{i} t_{i}$. It follows that the approximation ratio is at most $n$ and that it can be arbitrarily close to $n$ (when for example one value is 1 and the other $n-1$ values are arbitrarily high).

It is natural to ask whether there are better mechanisms than the proportional mechanism. In the next subsection, we give a positive answer by designing an optimal mechanism, albeit with not substantially better approximation ratio. 


\subsection{An optimal truthful mechanism}

In this subsection, we study truthful algorithms that have optimal approximation ratio.

To find an optimal truthful mechanism, we want to find functions $p_{i}(t)$ such that for every $t$ :

- for every $i: t_{i} p_{i}(t)$ is non-decreasing in $t_{i}$,

- for every $i: p_{i}(t)$ is non-increasing in $t_{i}$,

- $\sum_{i} p_{i}(t)=1$

and which minimize $\max _{t} \sum_{i} t_{i} p_{i}(t) / \min _{i} t_{i}$. The first two conditions capture truthfulness and the third condition the natural property that the probabilities add to 1 .

We will show the following theorem:

Theorem 1. There is a truthful in expectation mechanism without payments with approximation ratio $(n+1) / 2$. Conversely, no truthful in expectation mechanism without payments can have approximation ratio better than $(n+1) / 2$.

Before proceeding with the proof of the theorem, it is instructive to consider first the case of $n=2$ players. We will consider a symmetric mechanism, so it suffices to give the probabilities $p_{i}(t)$ of assigning the task to player $i$ when $t_{1} \leq t_{2}$. We claim that the mechanism with probabilities

$$
p_{1}(t)=1-\frac{t_{1}}{2 t_{2}} \quad p_{2}(t)=\frac{t_{1}}{2 t_{2}}
$$

is truthful and has approximation ratio $3 / 2$. To clarify: these are the probabilities when $t_{1} \leq t_{2}$; by symmetry, we can compute the probabilities when the declared value of the second player is smaller than the declared value of the first player.

Let us verify that this mechanism is truthful. Specifically we want to show that the expected cost of player $i$ is minimized when he declares his true value; this must hold for every value $t_{-i}$ of the other player. Consider first player 1 (the one with true value less than the declared value of the other player).

- He has no reason to declare something less than $t_{1}$, because $p_{1}(t)$ is non-increasing in $t_{1}$, consequently $t_{i} p_{i}(t) \leq t_{i} p_{i}\left(\tilde{t}_{i}, t_{-i}\right)$.

- He has no reason to declare something in $\left(t_{1}, t_{2}\right)$, because $t_{1} p_{1}(t)=t_{1}-\frac{t_{1}^{2}}{2 t_{2}}=$ $\frac{t_{2}^{2}-\left(t_{2}-t_{1}\right)^{2}}{2 t_{2}}$ is increasing in $t_{1}$ for $t_{1}<t_{2}$.

- Finally, he has no reason to declare something in $\left[t_{2}, \infty\right)$. In this case, his lie changes the order of the values, and, by the definition of the mechanism, the probability of getting the task will be $p_{2}\left(t_{2}, \tilde{t}_{1}\right)$. Nevertheless, we still have

$$
t_{1} p_{1}(t)=\frac{t_{2}^{2}-\left(t_{2}-t_{1}\right)^{2}}{2 t_{2}} \leq \frac{t_{2}}{2}=\tilde{t}_{1} \frac{t_{2}}{2 \tilde{t}_{1}}=\tilde{t}_{1} p_{2}\left(t_{2}, \tilde{t}_{1}\right) .
$$


We work similarly for the second player (the one with true value greater than the declared value of the other player). If he declares his true value $t_{2}$, his expected cost is $t_{2} p_{2}(t)=t_{1} / 2$.

- He has no reason to declare something less than $t_{1}$, because in this case the probability $p_{1}\left(t_{1}, \tilde{t}_{2}\right)$ of getting the task is at least $1 / 2$ and his cost will be at least $t_{2} / 2 \geq t_{1} / 2$.

- He has no reason to declare any other value greater than $t_{1}$ because his cost is going to be $\tilde{t}_{2} p_{2}\left(t_{1}, \tilde{t}_{2}\right)=t_{1} / 2$, anyway.

The above mechanism has approximation ratio $3 / 2$, because the cost is

$$
t_{1} p_{1}(t)+t_{2} p_{2}(t)=t_{1}-\frac{t_{1}^{2}}{2 t_{2}}+\frac{t_{1}}{2}=\frac{3}{2} t_{1}-\frac{t_{1}^{2}}{2 t_{2}} \leq \frac{3}{2} t_{1}
$$

Trivially, the approximation ratio tends to $3 / 2$ as $t_{2}$ tends to $\infty$.

We proceed to generalize the above to more than two players. Again, we define a symmetric mechanism, so it suffices to describe it when $t_{1} \leq \cdots \leq t_{n}$ :

$$
\begin{aligned}
& p_{1}=\frac{1}{t_{1}} \int_{0}^{t_{1}} \prod_{i=2}^{n}\left(1-\frac{y}{t_{i}}\right) d y \\
& p_{k}=\frac{1}{t_{k} t_{1}} \int_{0}^{t_{1}} \int_{0}^{y} \prod_{i=2 . . n, i \neq k}\left(1-\frac{x}{t_{i}}\right) d x d y \quad \text { for } k \neq 1
\end{aligned}
$$

For example, for $n=2$ we get the mechanism discussed above, and for $n=3$ the probabilities are

$$
p_{1}=1-\frac{t_{1}\left(t_{2}+t_{3}\right)}{2 t_{2} t_{3}}+\frac{t_{1}^{2}}{3 t_{2} t_{3}} \quad p_{2}=\frac{t_{1}}{2 t_{2}}-\frac{t_{1}^{2}}{6 t_{2} t_{3}} \quad p_{3}=\frac{t_{1}}{2 t_{3}}-\frac{t_{1}^{2}}{6 t_{2} t_{3}} .
$$

This definition is not arbitrary, but it is the natural solution to the requirements at the beginning of this subsection. This will become apparent as we proceed to show that this is an optimal algorithm for our problem.

First we verify that the mechanism is well-defined: We need to show that these probabilities are nonnegative and add up to 1 . Indeed, consider the quantities $q_{i}$ inside the integrals

$$
\begin{aligned}
& q_{1}(y)=\prod_{i=2}^{n}\left(1-\frac{y}{t_{i}}\right) \\
& q_{k}(y)=\frac{1}{t_{k}} \int_{0}^{y} \prod_{i=2 . . n, i \neq k}\left(1-\frac{x}{t_{i}}\right) d x
\end{aligned}
$$

for which

$$
p_{i}=\frac{1}{t_{1}} \int_{0}^{t_{1}} q_{i}(y) d y
$$

Since the integral is for $y \leq t_{1}$ and $t_{1}$ is the minimum among the $t_{k}$ 's, all factors in these expressions are nonnegative. This shows that $q_{i}(y) \geq 0$ for every $i=1, \ldots, n$. We also 
observe that $q_{1}^{\prime}(y)=-\sum_{i=2}^{n} q_{k}^{\prime}(y)$ which shows that $\sum_{i=1}^{n} q_{i}(y)$ is constant; taking $y=0$, we see that this constant is 1 . In summary the $p_{i}$ 's are nonnegative and their sum is $\frac{1}{t_{1}} \int_{0}^{t_{1}} \sum_{i=1}^{n} q_{i}(y) d y=\frac{1}{t_{1}} \int_{0}^{t_{1}} 1 d y=1$.

We also need to verify that this indeed a symmetric mechanism; otherwise the above definition is not complete. Specifically, we need to show that if $t_{1}=t_{2}$ then $p_{1}=p_{2}$. Indeed, it is straightforward to verify it by employing the following easy identity for every function $g$ :

$$
\int_{0}^{a}(a-y) g(y) d y \leq \int_{0}^{a} \int_{0}^{y} g(x) d x d y{ }^{2}
$$

In this case, $g(y)=\prod_{i=3}^{n}\left(1-\frac{y}{t_{i}}\right)$.

We now proceed to establish that the mechanism is truthful.

Lemma 1. The symmetric mechanism defined by the probabilities in (1) is truthful.

Proof. To show that the algorithm is truthful, we observe that

- The probabilities $p_{i}$ are non-increasing in $t_{i}$. This is trivially true for $i \neq 1$ and it can be easily verified for $i=1$. The fact that the probabilities are non-increasing in $t_{i}$ shows that no player $i$ has a reason to lie and declare a value $\tilde{t}_{i}<t_{i}$. To see this, fix the values of the remaining players and assume that player $i$ changes his value to $\tilde{t}_{i}<t_{i}$. We will argue that the probability of getting the task with the new value is greater than or equal to the original probability; this suffices because the cost of the player is this probability times $t_{i}$.

If, after the change, the order of the players remains the same, the probability does not decrease by this change because $p_{i}$ is non-increasing in $t_{i}$. We need to show the same when the change affects the order of the players. This turns out to be easy but we note that we need to be careful, because the expressions that define the probabilities of the mechanism depend on the order of the values. However, we don't need the expressions of the probabilities but a simpler argument: imagine that we lower the value from $t_{i}$ to $\tilde{t}_{i}$ in stages: from $t_{i}$ to $t_{i-1}$ to $t_{i-2}$, and so on, to $t_{i-k}$, and finally to $\tilde{t}_{i}$. In each stage, the order of the values remains the same and therefore the probability can only increase. It follows that it does not decrease from the total change. The fact that the mechanism is symmetric is crucial in this argument because the algebraic expressions of the probability of the player may change from stage to stage, but the values at the boundaries $t_{i-1}, \ldots, t_{i-k}$ of successive stages are the same.

- every player $k$ for $k \neq 1$ is truthful since $t_{k} p_{k}$ is independent of his value $t_{k}$; this holds even when the player reports a higher value which may change the order of the players

\footnotetext{
${ }^{2}$ Proof: Let $G(y)=\int_{0}^{y} g(x) d x$. Then

$\int_{0}^{a}(a-y) g(y)-\int_{0}^{a} \int_{0}^{y} g(x) d x d y=\int_{0}^{a}(a-y) g(y)-G(y) d y=\int_{0}^{a} a g(y)-(y G(y))^{\prime} d y=a G(a)-a G(a)=0$.
} 
- player 1 has no reason to lie and report a value in $\left(t_{1}, t_{2}\right]$ because $t_{1} p_{1}$ is increasing in $t_{1}$; furthermore, player 1 has no reason to report a higher value than $t_{2}$ which will change the order of the players because the cost will change from $t_{1} p_{1}$ to $t_{k}^{\prime} p_{k}^{\prime}=\frac{1}{t_{2}} \int_{0}^{t_{2}} \int_{0}^{y} \Pi_{i=3}^{n}\left(1-\frac{x}{t_{i}}\right) d x d y$ (because now the minimum value is $t_{2}$ ). It suffices therefore to show

$$
\begin{aligned}
& t_{1} p_{1} \leq t_{k}^{\prime} p_{k}^{\prime} \\
& \int_{0}^{t_{1}} \prod_{i=2}^{n}\left(1-\frac{y}{t_{i}}\right) d y \leq \frac{1}{t_{2}} \int_{0}^{t_{2}} \int_{0}^{y} \prod_{i=3}^{n}\left(1-\frac{x}{t_{i}}\right) d x d y \quad \Leftarrow \\
& \int_{0}^{t_{2}} \prod_{i=2}^{n}\left(1-\frac{y}{t_{i}}\right) d y \leq \frac{1}{t_{2}} \int_{0}^{t_{2}} \int_{0}^{y} \prod_{i=3}^{n}\left(1-\frac{x}{t_{i}}\right) d x d y \quad \Leftrightarrow \\
& \int_{0}^{t_{2}}\left(t_{2}-y\right) \prod_{i=3}^{n}\left(1-\frac{y}{t_{i}}\right) d y \leq \int_{0}^{t_{2}} \int_{0}^{y} \prod_{i=3}^{n}\left(1-\frac{x}{t_{i}}\right) d x d y
\end{aligned}
$$

The last holds because of Equation (2).

Putting everything together, we see that the mechanism is indeed truthful.

Lemma 2. The symmetric mechanism defined by the probabilities in (1) has approximation ratio $(n+1) / 2$.

Proof. Now that we have established that the algorithm is truthful, we proceed to bound its approximation ratio. The approximation ratio is $\sum_{i=1}^{n} t_{i} p_{i} / t_{1}$. Clearly, $t_{1} p_{1} \leq$ $t_{1}$ and for $k>1$

$$
\begin{aligned}
t_{k} p_{k} & =\frac{1}{t_{1}} \int_{0}^{t_{1}} \int_{0}^{y} \prod_{i=2 . . n, i \neq k}\left(1-\frac{x}{t_{i}}\right) d x d y \\
& \leq \frac{1}{t_{1}} \int_{0}^{t_{1}} \int_{0}^{y} 1 d x d y \\
& =t_{1} / 2 .
\end{aligned}
$$

Therefore, $\sum_{i=1}^{n} t_{i} p_{i} \leq t_{1}+(n-1) t_{1} / 2=t_{1}(n+1) / 2$, which shows that the approximation ratio is at most $(n+1) / 2$. It is trivial that if we fix the other values and let $t_{1}$ tend to 0 , the above inequalities are almost tight, and therefore the approximation ratio can be arbitrarily close to $(n+1) / 2$.

We will now show that no other truthful algorithm has a better approximation ratio.

Lemma 3. No truthful in expectation mechanism without payments has approximation ratio smaller than $(n+1) / 2$.

Proof. We will employ instances with values of the form $(1,1, m, \ldots, m)$ and $(1, m, \ldots, m)$ where $m$ is some large value (which we will allow to tend to infinity to obtain the lower bound). Without loss of generality we can assume that the mechanism is symmetric: if not, consider a non-symmetric mechanism $M$ and create a new mechanism which first permutes the values and then applies mechanism $M$; the new mechanism has approximation ratio smaller or equal to the approximation ratio of $M$. Let $p$ and 
$p^{\prime}$ be the probabilities assigned by an algorithm to the above instances. For the instance $(1,1, m, \ldots, m)$, we have $2 p_{2}+(n-2) p_{3}=1$ and the approximation ratio is at least $r \geq 2 p_{2}+m(n-2) p_{3}=m-2 p_{2}(m-1)$. Similarly for the other instance $(1, m, \ldots, m)$ we have $p_{1}^{\prime}+(n-1) p_{2}^{\prime}=1$ and $r \geq p_{1}^{\prime}+(n-1) m p_{2}^{\prime}=1+(m-1)(n-1) p_{2}^{\prime}$.

The crucial step is to use the truthfulness of player 2 to connect the two instances. Specifically, the second player is truthful only when $1 \cdot p_{2} \leq m \cdot p_{2}^{\prime}$. Substituting the value of $p_{2}^{\prime}$ in the bound of the second instance, we get that the approximation is at least $1+p_{2}(m-1)(n-1) / m$.

In summary, the approximation ratio is at least $\max \left\{m-2 p_{2}(m-1), 1+p_{2}(m-1)(n-\right.$ $1) / m$. The first expression is decreasing in $p_{2}$ while the second one is increasing in $p_{2}$; the minimum approximation ratio is achieved when the expressions are equal, that is, when $p_{2}=m /(2 m+n-1)$ which gives ratio at least $(n+1) / 2-\left(n^{2}-1\right) /(4 m+2 n-2)$. As $m$ tends to infinity, the approximation ratio tends to $(n+1) / 2$.

\section{Extension to many tasks and discussion}

In the previous section, we gave an optimal mechanism for one task. We can use it to get a mechanism for many tasks by running it independently for every task. The resulting mechanism is again truthful. If the objective is the social cost (i.e., to minimize the sum of the cost of all players), the mechanism clearly retains its approximation ratio. If the objective however is the makespan, then the approximation ratio is at most $n(n+1) / 2$, for the simple reason that $\max _{i} c_{i} \leq \sum_{i} c_{i} \leq n \max _{i} c_{i}$. So we get

Theorem 2. There is a truthful in expectation mechanism without payments for the problem of scheduling unrelated machines with approximation ratio $(n+1) / 2$ when the objective is the social cost and $n(n+1) / 2$ when the objective is the makespan.

It follows from the case of one task that the approximation ratio $(n+1) / 2$ is tight for the social cost. It is not clear that the pessimistic way we used to bound the approximation ratio of the makespan is tight. It remains open to estimate the approximation ratio of the given mechanism for many tasks. It is also open whether there are (task-independent or not) mechanisms without payments for many tasks with better approximation ratio.

\section{References}

[1] N. Alon, M. Feldman, A.D. Procaccia, and M. Tennenholtz. Strategyproof approximation of the minimax on networks. Mathematics of Operations Research, 35(3):513-526, 2010.

[2] V. Auletta, R. De Prisco, P. Penna, and G. Persiano. The power of verification for one-parameter agents. Automata, Languages and Programming, pages 63-82, 2004.

[3] G. Christodoulou, E. Koutsoupias, and A. Kovács. Mechanism design for fractional scheduling on unrelated machines. Automata, Languages and Programming, pages 40-52, 2007. 
[4] S. Dughmi and A. Ghosh. Truthful assignment without money. In Proceedings of the 11th ACM conference on Electronic commerce, pages 325-334. ACM, 2010.

[5] D. Fotakis and C. Tzamos. Winner-imposing strategyproof mechanisms for multiple facility location games. WINE, pages 234-245, 2010.

[6] A. Gibbard. Manipulation of voting schemes: a general result. Econometrica: Journal of the Econometric Society, pages 587-601, 1973.

[7] M. Guo and V. Conitzer. Strategy-proof allocation of multiple items between two agents without payments or priors. In Proceedings of the 9th International Conference on Autonomous Agents and Multiagent Systems: volume 1-Volume 1, pages 881-888. International Foundation for Autonomous Agents and Multiagent Systems, 2010.

[8] E. Koutsoupias and A. Vidali. A lower bound of $1+\varphi$ for truthful scheduling mechanisms. Mathematical Foundations of Computer Science 2007, pages 454-464, 2007.

[9] P. Lu, X. Sun, Y. Wang, and Z.A. Zhu. Asymptotically optimal strategy-proof mechanisms for two-facility games. In Proceedings of the 11th ACM conference on Electronic commerce, pages 315-324. ACM, 2010.

[10] P. Lu, Y. Wang, and Y. Zhou. Tighter bounds for facility games. WINE, pages 137$148,2009$.

[11] A. Mu'alem and M. Schapira. Setting lower bounds on truthfulness: extended abstract. In Proceedings of the eighteenth annual ACM-SIAM symposium on Discrete algorithms, pages 1143-1152. Society for Industrial and Applied Mathematics, 2007.

[12] N. Nisan and A. Ronen. Algorithmic mechanism design (extended abstract). In Proceedings of the thirty-first annual ACM symposium on Theory of computing, pages 129-140. ACM, 1999.

[13] N. Nisan, T. Roughgarden, E. Tardos, and V. Vazirani. Algorithmic game theory. Cambridge Univ Pr, 2007.

[14] K. Nissim, R. Smorodinsky, and M. Tennenholtz. Approximately optimal mechanism design via differential privacy. Arxiv preprint arXiv:1004.2888, 2010.

[15] A.D. Procaccia and M. Tennenholtz. Approximate mechanism design without money. In Proceedings of the tenth ACM conference on Electronic commerce, pages 177-186. ACM, 2009.

[16] K. Roberts. The characterization of implementable choice rules. Aggregation and Revelation of Preferences, pages 321-348, 1979.

[17] M.A. Satterthwaite. Strategy-proofness and Arrow's conditions: Existence and correspondence theorems for voting procedures and social welfare functions. Journal of Economic Theory, 10(2):187-217, 1975. 